\title{
Nationale NCD-Strategie: Der Mensch steht im Zentrum
}

\section{Christine Romann}

Dr. med., Mitglied des Zentralvorstandes der FMH, Departementsverantwortliche Gesundheitsförderung und Prävention

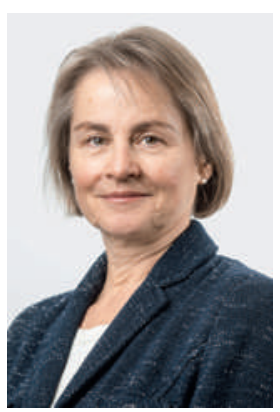

Im Herbst 2013 hatte der Dialog Nationale Gesundheitspolitik - die ständige Plattform von Bund und Kantonen zu aktuellen gesundheitspolitischen Themen - die Weichen gestellt für die Erarbeitung einer nationalen Strategie zur Prävention nichtübertragbarer Krankheiten (noncommunicable diseases $=$ $N C D s) . \mathrm{Zu}$ diesen zählen gemäss WHO Diabetes, Herz-Kreislauf-Erkrankungen, Krebs, chronische Atemwegserkrankungen und psychische Störungen. In der Schweiz werden auf Grund der Häufigkeit und Krankheitslast die muskuloskelettalen Erkrankungen dazugezählt; hingegen sind die psychischen Störungen nicht Teil der NCD-Strategie, sondern werden im Dialogprojekt «Psychische Gesundheit» gesondert angegangen.

Die NCDs sind für $86 \%$ aller Todesfälle und $77 \%$ der Krankheitslast in der Europäischen Region der WHO verantwortlich [1]. Charakteristisch für diese NCDs sind ihre gemeinsamen Risikofaktoren und Determinanten, aus welchen sich NCD-übergreifende Präventionschancen ergeben - diese gilt es nun zu nutzen! Die nun erstmals vorgeschlagene nationale NCD-Strategie wurde von einem erweiterten Leitungsgremium entwickelt: Die Grundlagen dazu entstanden in zwei Arbeitsgruppen unter Einbezug der Stakeholder, darunter auch der FMH, die ebenfalls im erweiterten Leitungsgremium Einsitz nehmen konnte. Der Entwurf dieses Strategiepapiers geht nun in eine breite Vernehmlassung. Wir stellen hier die wichtigsten Inhalte vor:

\section{Nichtübertragbare Krankheiten sind für $86 \%$ der Todesfälle und 77\% der Krankheitslast verantwortlich.}

Der einzelne Menschen steht im Zentrum der Überlegungen, die Vision nimmt diesen Aspekt folgendermassen auf: "Mehr Menschen bleiben unabhängig ihres sozioökonomischen Status gesund oder haben trotz chronischer Krankheit eine hohe Lebensqualität. Weniger Menschen erkranken an vermeidbaren nichtübertragbaren Krankheiten oder sterben vorzeitig. Die Bevölkerung ist befähigt, gesundheitsförderliche Lebenswelten zu gestalten sowie einen gesunden Lebensstil zu pflegen.»
Mit einer Kombination aus Verhaltensprävention - bei der vorab Menschen mit einem erhöhten Risiko im Fokus sind - und Verhältnisprävention - mit der gesundheitsförderndes Verhalten erleichtert und krankmachendes Verhalten erschwert wird - soll dieses Ziel

Der Prävention kommt deshalb ein hoher Stellenwert zu: Notwendig hierfür sind sowohl strukturelle Voraussetzungen als auch eine geregelte Vergütung.

erreicht werden. In der Verhaltensprävention kommt der Integration der Prävention in die medizinische Versorgung eine hohe Bedeutung zu! Dafür braucht es strukturelle Voraussetzungen und eine geregelte Vergütung aller präventiven Leistungen der dazu befugten Leistungserbringer. Immer wenn Menschen mit dem Gesundheitsversorgungssystem in Berührung kommen, soll die Chance genutzt werden, sie für Präventionsthemen zu sensibilisieren und ihnen gegebenenfalls bei ihren Präventionsbemühungen behilflich zu sein, wie dies zum Beispiel mit dem Projekt des KHM «Gesundheitscoaching» vorbildlich bereits geschieht. Die Prävention in der Gesundheitsversorgung ist ein Angebot - das auch ausgeschlagen werden kann: Die Selbstbestimmung der bzw. des Einzelnen muss immer respektiert werden, nur so kann Prävention in der Gesundheitsversorgung erfolgreich sein!

Der Strategieentwurf stützt sich auf die beiden umfangreichen und sehr sorgfältig ausgeführten Arbeitsgruppenberichte, die jeweils von einer von der Arbeitsgruppe bestimmten Redaktionskommission verfasst wurden und die intensive, etwa anderthalbjährige Arbeit der in den Arbeitsgruppen vertretenen Stakeholder sehr gut reflektieren. Die Berichte gehen bereits deutlich weiter als der nun vorliegende Strategieentwurf und es ist $\mathrm{zu}$ hoffen, dass sie auch die 2016 geplante Erarbeitung der Massnahmenpakete inspirieren werden. Denn diese und ihre Umsetzung werden entscheidend dafür sein, ob das anvisierte Ziel erreichbar ist. Der Strategieentwurf ist nun zunächst ein guter Schritt in die richtige Richtung!

1 www.euro.who.int/en/health-topics/noncommunicable-diseases 